\title{
Adenovirus-mediated combined anti-angiogenic and pro-apoptotic gene therapy enhances antitumor efficacy in hepatocellular carcinoma
}

\author{
FEI YAN ${ }^{1,2}$, YI ZHENG ${ }^{1}$ and LAIQIANG HUANG ${ }^{1}$ \\ ${ }^{1}$ The Shenzhen Key Lab of Gene and Antibody Therapy, Center for Biotech and Bio-Medicine and Division of Life Sciences, \\ Graduate School at Shenzhen, Tsinghua University; ${ }^{2}$ Paul C. Lauterbur Research Center for Biomedical Imaging, \\ Institute of Biomedical and Health Engineering, Shenzhen Institutes of Advanced Technology, \\ Chinese Academy of Sciences, Shenzhen 518055, P.R. China
}

Received July 1, 2012; Accepted September 10, 2012

DOI: $10.3892 / \mathrm{ol} .2012 .987$

\begin{abstract}
A previous study reported that combinatorial human endostatin and soluble tumor necrosis factor (TNF)-related apoptosis-inducing ligand (sTRAIL) gene transfer suppresses human hepatocellular carcinoma (HCC) growth and angiogenesis using the pVAX1 plasmid vector. The current study investigated the antitumor efficacy in HCC through adenovirus-mediated combination gene therapy. Human endostatin and sTRAIL (114 to 281 AA) genes were amplified and cloned into the Adeno-X expression vector. The recombinant adenoviruses (Ad-E and Ad-T) were packaged, amplified in the HEK 293 cells and used to infect human umbilical vein endothelial cells (HUVECs) and HepG2 cells, respectively. The results revealed that a significant cell growth inhibition was observed in the two types of cells using a cell viability assay. Intratumoral administration with Ad-E and Ad-T revealed a significant enhanced regression of the tumors compared with treatment with either recombinant adenovirus alone. Histology and immunohistochemistry examination further indicated that the inhibition of tumor growth appeared to result from increased apoptosis and reduced angiogenesis in tumor xenografts. In conclusion, these data further confirm the enhancement of antitumor efficacy through combined endostatin and TRAIL gene therapy and provide a promising application prospect by virtue of adenovirus-mediated anti-angiogenic and pro-apoptotic cancer gene therapy.
\end{abstract}

Correspondence to: Professor Laiqiang Huang, The Shenzhen Key Lab of Gene and Antibody Therapy, Center for Biotech and Bio-Medicine and Division of Life Sciences, Graduate School at Shenzhen, Tsinghua University, Shenzhen 518055, P.R. China E-mail: huanglq@sz.tsinghua.edu.cn

Key words: hepatocellular carcinoma, cancer gene therapy, adenovirus, endostrain, tumor necrosis factor-related apoptosis-inducing ligand

\section{Introduction}

Hepatocellular carcinoma (HCC) is one of the most common types of primary cancer in the world (1), ranking the sixth most prevalent cancer and the third most frequent cause of cancer-related mortality (2). More than half a million individuals are diagnosed with this disease every year worldwide, including approximately 20,000 new cases in the USA (3). Hepatocellular carcinoma has a poor 5-year survival rate of approximately $7 \%$ despite treatment (4). Potentially curative therapies, including liver transplantation and surgical resection, are only applied to a minority of subjects due to the advanced stage of the disease at the time of diagnosis and the lack of suitable organ donors. Other regional treatments may be beneficial for unresectable HCC, but local failure or recurrences are frequent and the long term survival rate remains poor.

Gene therapy may offer a new therapeutic option for HCC and is considered to be a potential adjuvant of other therapies (5). Previous clinical trials have shown that the side-effects are acceptable in the majority of the cases and the mechanism of action is different from standard treatments (6). In the past decade, the gene therapy of liver cancer has covered a variety of gene transfer strategies aimed to treat patients with primary and secondary liver tumors, including gene-directed enzyme/pro-drug therapy, inhibition of oncogenes and restoration of tumor-suppressor genes, immunotherapy, antiangiogenesis and virotherapy (7). However, several of these strategies have reached early clinical development with little success. The main obstacles to further progress lie in the poor gene delivery efficiency and therapeutic effect of single genes. It is a widely accepted theory that cancer is a complex disease, with multiple genes involved in diverse pathways. Therefore, it may be possible to achieve a more effective control of tumor growth through selecting effective gene delivery approaches and an appropriate combination of therapeutic genes.

Tumor necrosis factor (TNF)-related apoptosis-inducing ligand (TRAIL) belongs to the TNF family (8), which functions as a cytokine to selectively induce apoptosis in cancer cells and has minimal or no toxicity against normal tissues 
in vitro and in vivo $(9,10)$. TRAIL is known to bind with TRAIL-R1 [death receptor (DR) 4], TRAIL-R2 (DR5), TRAIL-R3 [decoy receptor (DcR) 1], TRAIL-R4 (DcR2) and osteoprotegerin. Among these, TRAIL-R1 and -R2 possess intracellular death domains and, subsequently, have the ability to mediate TRAIL-induced apoptosis (11). Converseley, the immune defense role of TRAIL has been shown to kill pathogen-infected or malignant cells (12). Numerous studies have demonstrated the potential use of recombinant soluble TRAIL as a cancer therapeutic agent. The extracellular domain of TRAIL works as a soluble cytokine (sTRAIL) and induces apoptosis on cancer cells at distant locations from the producing cell (13).

Endostatin, a carboxyl-terminal proteolytic fragment of collagen XVIII, is a key tumor suppressor and has been used highly successfully to treat cancer. Endostatin has a multi-binding ability to tropomyosin, heparin, perlecan, glypican, integrin, zinc, laminin and fibulin (14). By virtue of these binding interactions and a variety of downstream effects, endostatin acts as a specific inhibitor of endothelial cell proliferation, migration and angiogenesis. This results in the inhibition of tumor growth $(15,16)$. Previous research has also demonstrated that endostatin may inhibit angiogenesis in hepatocellular carcinoma following transarterial chemoembolization (17).

Replication-defective adenoviral (Ad) vectors have shown promise as a tool for gene delivery-based therapeutic applications due to their high gene delivery efficiency. Moreover, adenoviruses have a number of other advantages as gene delivery vectors, including the ability to transduce a wide variety of non-dividing and dividing cells with high efficiency, relative ease of construction and ability to be purified as hightiter viral stocks. These characteristics make adenoviruses particularly attractive for overexpressing specific genes in vitro and for evaluating in vivo biological activity in animal models (18). Gendicine, the first gene therapy product approved by China FDA, is composed of the adenoviral vector and the human wild-type p53 tumor suppressor gene. Clinical trials have confirmed that it is safe and effective for head and neck squamous cell carcinoma (19).

Previous evidence has shown that carcinoma growth and angiogenesis were suppressed by combined plasmid-mediated endostatin and TRAIL gene therapy in mice (20). The current study reports on the use of an adenovirus as the gene delivery vector to construct the recombinant adenovirus AdenoX-TRAIL (Ad-T) and Adeno-X-endostatin (Ad-E) and to examine whether the combination of TRAIL with endostatin works synergistically against HCC.

\section{Materials and methods}

Cell lines and animals. The human embryonic kidney HEK293 cells, human hepatocellular carcinoma HepG2 cells and human umbilical vein endothelial cells (HUVECs) were obtained from the American Type Culture Collection (ATCC, Rockville, MD, USA) and maintained in DMEM (Life Technologies, Carlsbad, CA, USA) supplemented with $10 \%$ heat-inactivated fetal bovine serum (Life Technologies) and antibiotics. For the HUVECs, $10 \mathrm{ng} / \mathrm{ml}$ VEGF was added to the media. The culture was maintained in a $95 \%$ air humidified atmosphere containing $5 \% \mathrm{CO}_{2}$ at $37^{\circ} \mathrm{C}$. Specific pathogen-free six-week-old female BALB/c nude mice were obtained from Beijing Weitong Lihua Test Animal Co. (Beijing, China). All animals were housed under pathogen-free conditions. The animal experiments were carried out according to the Institutional Guidelines of Tsinghua University Graduate School at Shenzhen.

Construction of recombinant adenovirus. Adeno-X expression system (BD Clontech, Mountain View, CA, USA) was used to construct recombinant adenovirus vectors. Complementary DNA encoding 184 amino acid residues of human endostatin and 167 amino acid residues of human TRAIL (from 114 to 281 amino acids), including the signal peptide of human interleukin-2, were PCR-amplified from pMD-18-endostatin and pMD-18-TRAIL and subcloned into the pShuttle plasmids. The expression cassettes, including the cytomegalovirus (CMV) promoter, endostatin or sTRAIL gene fragments and bovine growth hormone polyadenylation (BGH) polyA tails, were then removed from pShuttleendostatin and pShuttle-TRAIL by PI-SceI and I-CeuI digestion and inserted into the corresponding PI-Sce I and I-Ceu I sites of linearized adenoviral backbone. Thus two recombinant adenoviral plasmids (named Ad-E and Ad-T) were obtained. The correct recombinants were selected and retransformed into DH5 $\alpha$-competent cells. Purified recombinant plasmids were linearized by $\mathrm{PacI}$ restriction and transfected into HEK293 cells to generate recombinant adenoviruses. Recombinant viruses were propagated in HEK293 cells, purified using an Adeno-X ${ }^{\mathrm{TM}}$ Maxi purification kit and kept in a solution containing $10 \%$ glycerol, $10 \mathrm{mM}$ Tris (pH 7.6) and $1 \mathrm{mM} \mathrm{MgCl}_{2}$. Ad-EGFP was used as a control adenovirus expressing an enhanced green fluorescent protein reporter gene. Viral titers were calculated by determination of the TCID50 or with the use of the adeno-X rapid titer kit (BD Bioscience, Palo Alto, CA, USA). Titers are expressed as either multiplicity of infection (MOI) or as plaque-forming units $(\mathrm{pfu}) / \mathrm{ml}$.

Western blot analysis to detect endostatin and STRAIL expression. HUVECs and HepG2 cells were seeded in 6-well plates and grown to $80 \%$ confluence, at which point the culture medium was replaced with serum-free medium and the cells were infected with recombinant adenoviruses at an MOI of 10 or 50 . The media were collected $48 \mathrm{~h}$ later and the concentrated conditioned supernatants were subjected to western blot analysis in a standard procedure. Ad-EGFP was used for the visual examination of transgene expression under fluorescent microscope.

Cell viability assay. Cell viability was determined by MTT assay. In brief, $2 \times 10^{4}$ HUVECs and HepG2 cells were seeded in 96-well plates and infected with Ad-E or Ad-T, respectively, at either 10 or $50 \mathrm{MOI}$. At 24, 48 or $72 \mathrm{~h}$ following virus infection, $10 \mu \mathrm{l}$ of $5 \mathrm{mg} / \mathrm{ml}$ MTT in PBS solution was added into the media and incubated for a further $4 \mathrm{~h}$. Subsequently, the formazan product was solubilized by addition of $100 \mu \mathrm{l}$ of dimethyl sulfoxide. Absorbance was measured at a wavelength of $490 \mathrm{~nm}$ using a microplate reader and cellular viability (\%) was determined. 
A
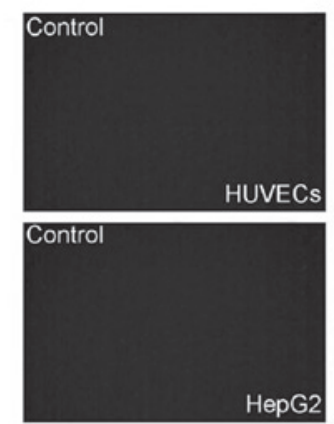

$\mathbf{B}$
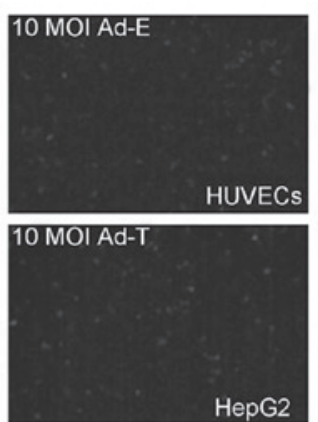
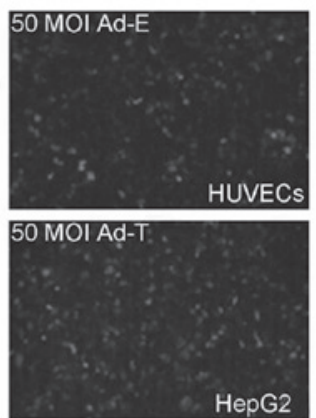

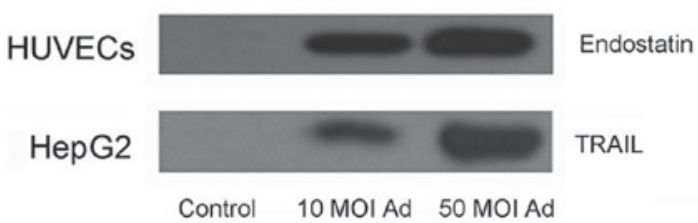

Figure 1. Expression of recombinant proteins $48 \mathrm{~h}$ following infection in HepG2 cells and HUVECs. The cells were infected with 10 or 50 MOI viruses (A) The expression of green fluorescent protein in HUVECs (upper row) and HepG2 cells (lower row) was visualized under a fluorescent microscope. (B) The expression of endostatin and TRAIL in HUVEC (upper row) and HepG2 cell (lower row) supernatants was detected using western blotting analysis. TRAIL, tumor necrosis factor-related apoptosis-inducing ligand; HUVEC, human umbilical vein endothelial cell; MOI, multiplicity of infection.

Animal experiments. All animal studies were approved by our Institute's Animal Care and Use Committee. HepG2 cells $\left(1 \times 10^{7}\right)$ were injected into the right flank of female $\mathrm{BALB} / \mathrm{c}$ nude mice in $200 \mu \mathrm{l}$ serum-free medium at the age of 6-8 weeks. Therapy was initiated when mice had developed palpable tumors with diameters of 5-8 mm 10 days following inoculation. Mice were randomly divided into 5 groups with 5 animals each and subjected to intratumoral injections of virus suspension as follows: Group 1, $100 \mu \mathrm{l}$ PBS (PBS group); Group 2, $100 \mu \mathrm{l}$ Ad-EGFP virus ( $2 \times 10^{9}$ pfu per tumor); Group

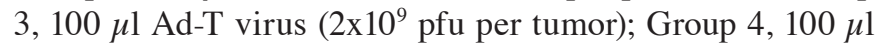
Ad-E virus ( $2 \times 10^{9}$ pfu per tumor) and Group 5, $100 \mu \mathrm{l} \mathrm{Ad-T}$ + Ad-E virus $\left(1 \times 10^{9}\right.$ pfu Ad-T and $1 \times 10^{9}$ pfu Ad-E per tumor). The treatment procedure was repeated three more times, at 3-day intervals. Tumor growth was monitored by caliper measurements twice a week and tumor volume was calculated using the formula: $0.52 \mathrm{x}$ (largest diameter $\mathrm{x}$ smallest diameter ${ }^{2}$ ). The tumors were extracted for histology and immunohistochemistry examination.

Histology and immunohistochemistry examination. Tumor-bearing mice were sacrificed one week following the last treatment and the tumors were dissected, covered with Tissue-Tek (Sakura Finetek Europe B.V., Alphen aan den Rijn, The Netherlands) and then frozen in liquid nitrogen vapor for further histological examination. Tumor sections $(5-\mu \mathrm{m}$ thick) were cut with a cryostat microtome (CM1950; Leica, Heidelberg, Germany) and stained with hematoxylin and eosin (HE) as described previously (21). Terminal deoxynucleotidyl transferase-mediated dUTP nick end labeling (TUNEL) staining was performed to quantitatively assess tumor apoptosis with an in situ Cell Death Detection kit (Roche Diagnostics, Mannheim, Germany) according to the manufacturer's instructions. The number of apoptotic cells was quantified by determining the percentage of positively stained cells for all nuclei from six randomly chosen fields/sections at x200 magnification. Tumor sections were also fixed in acetone, incubated and stained with an anti-CD31 antibody to detect tumor microvessel density (MVD) as previously reported (22). MVD was determined by counting the number of microvessels per high-power field.

Statistical analysis. Statistical analysis was carried out with SPSS software (version 13.0 for Windows). All values are expressed as mean \pm SD. Data were analyzed by one-way ANOVA and then differences among the means were analyzed using the Kaplan-Meier multiple comparison test. Differences were considered significant at $\mathrm{P}<0.05$.

\section{Results}

Expression detection of recombinant endostatin and TRAIL. Fluorescent microscope examination confirmed the expression of EGFP proteins in the HUVECs and HepG2 cells infected with Ad-EGFP virus (Fig. 1A). In order to examine whether endostatin and TRAIL genes inserted into the adenovirus vector were able to express and secrete, the supernatants of HUVECs and HepG2 cells infected with Ad-E or Ad-T was detected by western blotting using anti-endostatin and anti-TRAIL antibodies, respectively. Results revealed that an endostatin protein band of $20 \mathrm{kDa}$ and a TRAIL protein band of $18.5 \mathrm{kDa}$ were able to be detected, whereas no such band was present in the control supernatants of cells infected with Ad-EGFP virus. A higher level expression of in $50 \mathrm{MOI}$ infected cell supernatants than that of $10 \mathrm{MOI}$ infected cell supernatants following $48 \mathrm{~h}$ was also observed (Fig. 1B). These results confirmed the effective expression of transgenes by using Adeno-X expression system.

Cell viability assay. The HUVECs and HepG2 cells were infected with Ad-E and Ad-T, respectively, using 10 or 50 MOI. Ad-EGFP adenovirus vector served as a control. Fig. 2 shows cell growth inhibition of HUVECs and HepG2 cells treated with Ad-E and Ad-T virus following 24, 48 or 72 h, respectively. When HUVECs were infected with Ad-E 

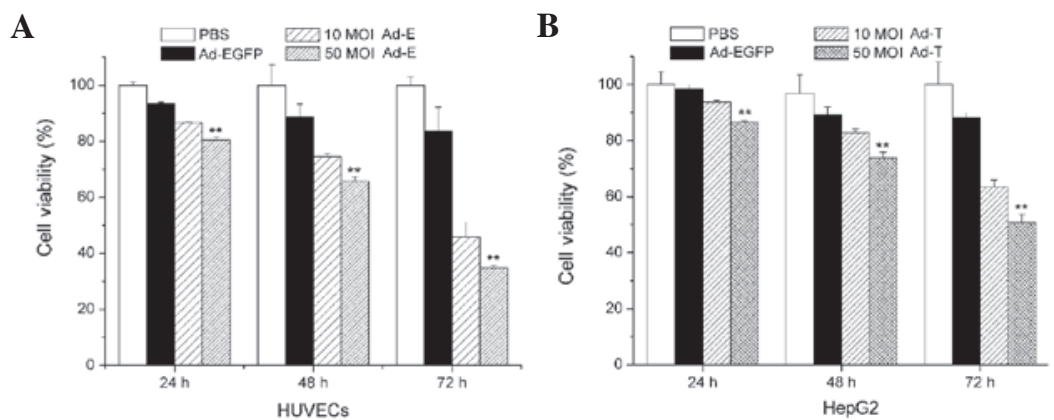

Figure 2. Cell viability of HepG2 cells and HUVECs. (A) HUVECs were infected with 10 MOI or 50 MOI Ad-E recombinant viruses for 24,48 or $72 \mathrm{~h}$. (B) HepG2 cells were infected with 10 MOI or 50 MOI Ad-T recombinant viruses for 24, 48 or $72 \mathrm{~h}$. HUVEC, human umbilical vein endothelial cell; MOI, multiplicity of infection.
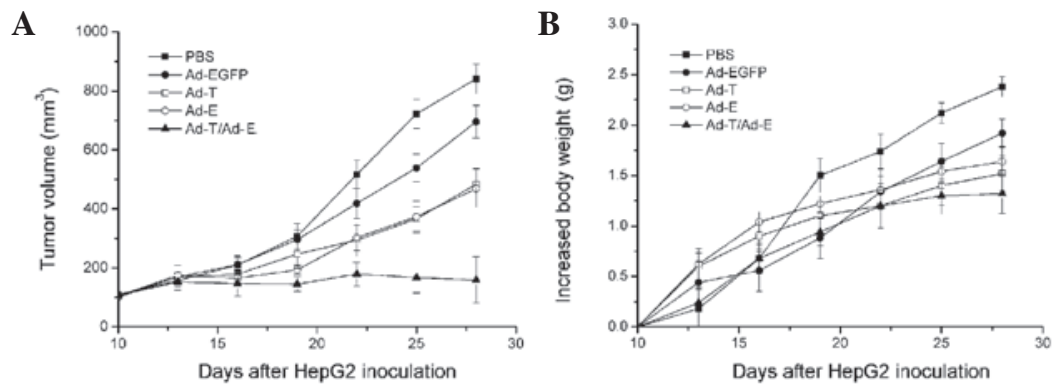

Figure 3. Tumor growth inhibition of the established tumor in BALB/c nude mice. Tumor-bearing mice were injected via intratumoral injection with PBS ( $\mathbf{})$,

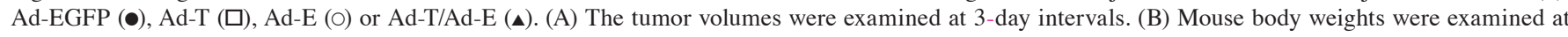
3-day intervals.
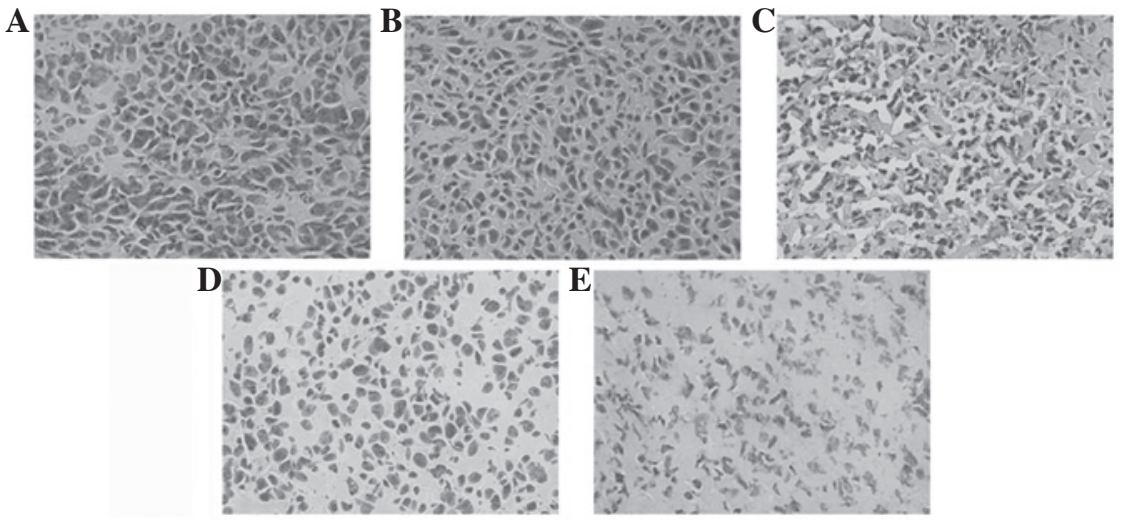

Figure 4. HE staining assay. Sections of tumors embedded from tumor-bearing mice treated with (A) PBS, (B) Ad-EGFP, (C) Ad-T, (D) Ad-E and (E) Ad-T/Ad-E recombinant virus. Sections were observed under a light microscope (x200). HE, hematoxylin and eosin.

at $10 \mathrm{MOI}$, the cell viability of HUVECs following 24,48 and $72 \mathrm{~h}$ was $93.74,82.85$ and $63.30 \%$, respectively. With the increasing amount of virus (50 MOI) added into the media, a significantly lower cell viability was observed, with 86.61 , 73.92 and $50.76 \%$ following 24,48 and $72 \mathrm{~h}$ virus infection $(\mathrm{P}<0.01$; Fig. 2A). Similarly, cell growth inhibition was also observed in the Ad-T-infected HepG2 cells. When HepG2 cells were infected with Ad-T at $10 \mathrm{MOI}$, the cell viability of HepG2 following 24, 48 and $72 \mathrm{~h}$ was $91.61,74.48$ and $45.83 \%$, respectively. A more significant inhibition of cell proliferation was observed when using 50 MOI Ad-T virus infection, achieving 86.61, 73.92 and 50.76\% following 24, 48 and $72 \mathrm{~h}$ virus infection $(\mathrm{P}<0.01$; Fig. $2 \mathrm{~B})$. Treatment with Ad-EGFP adenovirus vector had no significant cell growth inhibition effect on HUVECs and HepG2 cells at various time points.

In vivo antitumor efficacy of Ad-E and Ad-T. Subsequently, the efficacy of antitumor treatment with Ad-E and Ad-T was investigated (Fig. 3A). In control mice treated with only PBS, HepG2-derived tumors reached a volume of $841.47 \pm 50.32 \mathrm{~mm}^{3}$ by day 28 following implantation. There was no significant tumor inhibition effect when mice were treated with Ad-EGFP $\left(695.33 \pm 55.50 \mathrm{~mm}^{3}\right.$ tumor volume on day 28; $\mathrm{P}>0.05$ ). In comparison, the tumors treated with Ad-T or Ad-E were significantly smaller than those treated with PBS control, reaching only $483.48 \pm 52.28$ and $468.76 \pm 63.73 \mathrm{~mm}^{3}$, 


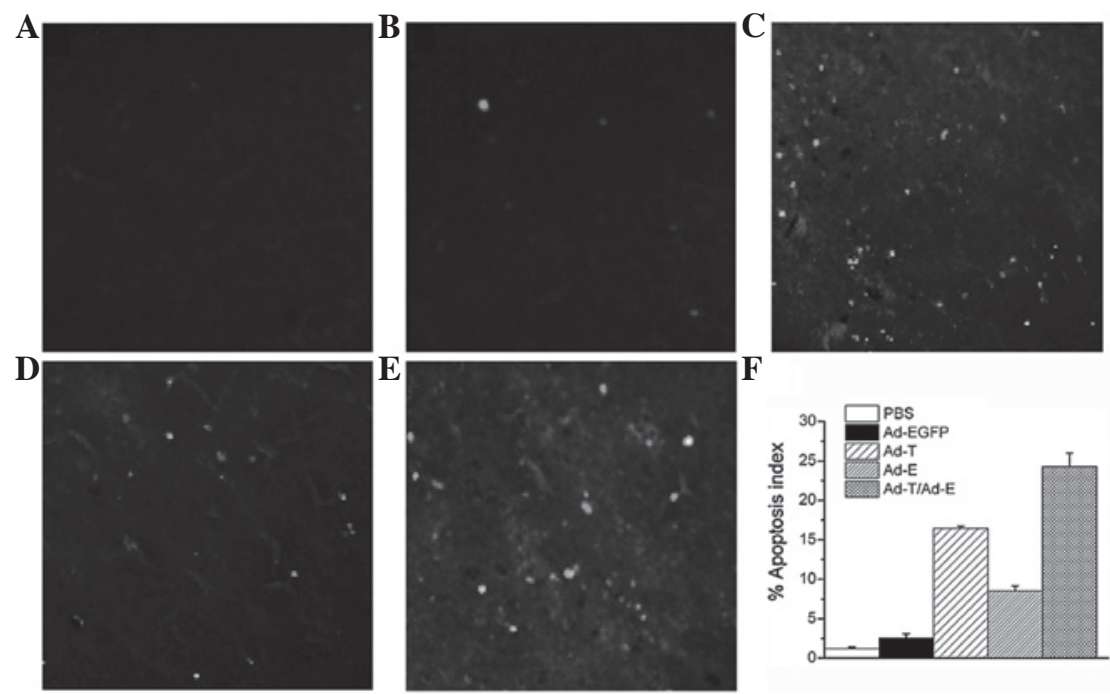

Figure 5. TUNEL assay of tumors. Sections of tumors embedded from tumor-bearing mice treated with (A) PBS, (B) Ad-EGFP, (C) Ad-T, (D) Ad-E and (E) Ad-T/Ad-E recombinant virus. Apoptotic nuclei were observed under a fluorescence microscope $(\mathrm{x} 200)$. (F) The apoptosis index. TUNEL, terminal deoxynucleotidyl transferase-mediated dUTP nick end labeling.

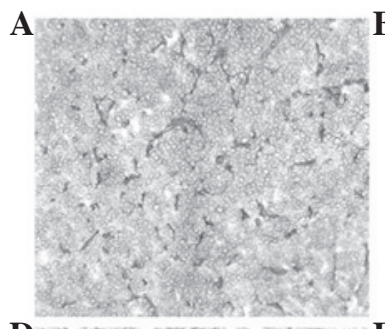

D

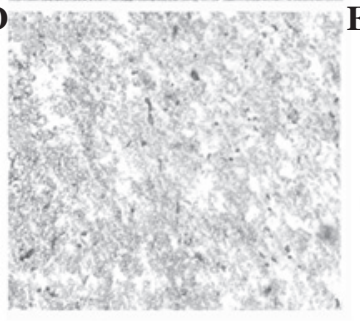

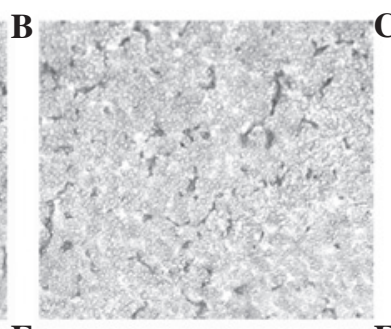

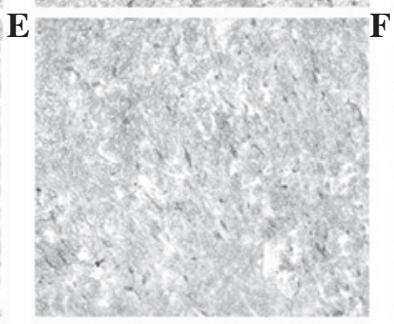

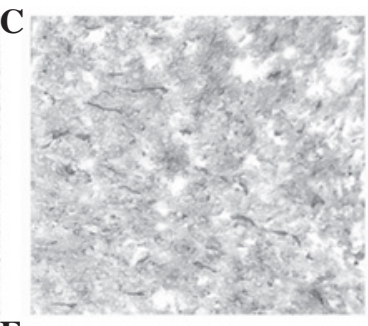

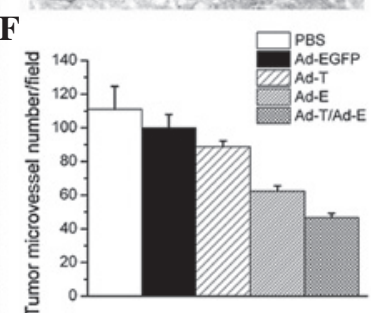

Figure 6. Immunohistochemical analysis of CD31 expression of tumors. Sections of tumors embedded from tumor-bearing mice treated with (A) PBS, (B) Ad-EGFP, (C) Ad-T, (D) Ad-E and (E) Ad-T/Ad-E recombinant virus. Positive cells were observed under a light microscope (x200). (F) The mean microvessel density index.

respectively $(\mathrm{P}<0.05)$. Notably, combined treatment with Ad-T + Ad-E resulted in a significant reduction in tumor volume $\left(158.72 \pm 78.30 \mathrm{~mm}^{3}\right)$, compared with all other groups on day 28 following implantation $(\mathrm{P}<0.01)$. Changes in body weight of mice demonstrated a similar trend (Fig. 3B). In the PBSand Ad-EGFP-treated groups, the mean increases in the body weight of mice by day 28 following implantation were $2.38 \pm 0.35$ and $2.09 \pm 0.28 \mathrm{~g}$, respectively. In comparison, the mean increase in body weight of mice treated with the single Ad-T virus was $1.64 \pm 0.24 \mathrm{~g}$ and that of mice treated with the single Ad-E virus was $1.52 \pm 0.15 \mathrm{~g}$. Other than that, combined treatment with Ad-E and Ad-T virus revealed the least increase in body weight of mice, with a $1.32 \pm 0.24 \mathrm{~g}$ mean increased body weight per mouse $(\mathrm{P}<0.01)$.

Histology and immunohistochemistry examination. Histologically, control tumors (PBS and Ad-EGFP) exhibited no or little tumor necrosis. Tumors treated with Ad-E or Ad-T demonstrated a significant tumor necrosis. However, tumors treated with Ad-E + Ad-T had the most significantly visible homogeneous necrosis with a clearly distinguishable morphology compared with controls (Fig. 4). A TUNEL assay confirmed that there were extremely few apoptotic cells in the control tumors (PBS and Ad-EGFP), with 1.18 \pm 0.21 and $2.52 \pm 0.54 \%$ apoptotic cells, respectively. Tumors treated with Ad-E and Ad-T had 8.52 and $16.45 \pm 0.28 \%$ apoptotic cells, respectively, but the majority of apoptotic cells were identified in the tumors treated with Ad-E + Ad-T, achieving 24.30 $\pm 1.68 \%$ apoptotic cell ratio (Fig. 5; $\mathrm{P}<0.01$ ). Angiogenesis in tumor tissues was estimated by MVD assay. As shown in Fig. 6, treatment with Ad-E + Ad-T resulted in a significant reduction in tumor MVD compared with the PBS-, Ad-EGFP-, Ad-E or Ad-T virus-treated groups $(\mathrm{P}<0.01)$. Quantitation revealed $46.76 \pm 2.51$ tumor microvessels per field in the tumors treated 
with Ad-E + Ad-T, which was significantly lower compared with the PBS (111.24 \pm 13.60 microvessels), Ad-EGFP (98.06 \pm 8.24 microvessels), Ad-T (88.67 \pm 3.52 microvessels) and Ad-E group (62.34 \pm 3.06 microvessels).

\section{Discussion}

HCC, similar to other malignant tumors, is a complex disease with multiple genes in diverse pathways involved in its initiation, progression, invasion and metastasis. It is widely accepted that the sequential accumulation of mutations that activate oncogenes and disrupt tumour suppressor genes, combined with multiple cycles of clonal selection and evolution, facilitate the process of carcinogenesis. Moreover, during the growth of a tumor, cancer metastasis may occur by virtue of a series of aggressive steps, including leaving the primary mass, intravasation, survival in circulation, extravasation and colonization and growth of tumor cells at a distant site. The promotion of tumor cell apoptosis and inhibition of angiogenesis provides a good chance of preventing cancer from overgrowing and becoming malignant $(23,24)$. TRAIL is regarded as one of the most potent inhibitors of tumor growth. Anti-angiogenic factors, including endostatin, have been identified and have demonstrated the ability to inhibit tumor growth in vivo (25). Therefore, a combination gene therapy of TRAIL and endostatin is likely to achieve an improved control of tumor growth through the combination of TRAIL-induced tumor cell apoptosis and endostatin-induced anti-angiogenesis, as demonstrated in the presented study (Fig. 3). The efficacy of enhancement of tumor treatment has been demonstrated by the combination of TRAIL or endostatin with chemotherapy and radiotherapy (26-29).

The key point of gene therapy is to establish an effective gene delivering system. Our data indicate that the combination gene therapy of adenovirus-mediated TRAIL with endostatin exhibits significant antitumor activities through induction of apoptosis and inhibition of angiogenesis, compared with single Ad-T or Ad-E. Our results are in agreement with the previous study by Zhang et al (20), in which sTRAIL and endostatin genes were transferred using plasmid pVAX1 as a vector. By contrast, the Adeno-X expression system was utilized in our study. The Adeno-X expression system offers a simple yet powerful method for constructing recombinant adenovirus by using a ligation-based method. The Adeno-X expression system is also considered as the most efficient adenoviral system available and generates high-level protein expression in a wide variety of mammalian host cells. Furthermore, viral vectors have been preferentially applied in gene therapy due to their marked advantages over the current plasmid-transfection methods, including i) the protocols are simple to perform, involving the direct addition of virus to cells; ii) the use of Ad-gene vectors does not require any additional reagents; and iii) observable adenoviral transduction efficiency is high and reproducible in a number of the cell types tested when used at an optimal MOI.

The current study revealed that the ratio of apoptotic cells significantly increased in the tumors treated with a combination of Ad-E and Ad-T virus, in comparison with signal gene treatment (Fig. 5). The enhanced cytotoxic sensitivity of tumor cells may be attributed to the following several factors. The overexpression of endostatin may result in the inhibition of angiogenesis and deprive cancer cells of the nutritional supplements that they require for sustaining their high metabolic activity. Thus, nutrient deficiency reprograms cancer cells to enter into apoptosis. Previous evidence has shown that nutrition deprivation causes cancer cell death (30). Braun et al demonstrated that serum-nutrient starvation induces cell death mediated by Bax and Puma (31). However, under the condition of nutrient/oxygen depletion, cancer cells are expected to be more sensitive towards an apoptotic inducer. Although there are reports that the majority of HCC cells are insensitive towards TRAIL-induced apoptosis, it is likely that HCC cells tend to be more sensitive to TRAIL-induced apoptosis when tumors are subjected to hypoxic and nutrient-depleted environments. In addition, it was also notable that intratumoral MVD significantly decreased in the residual tumors with combination therapy, compared with Ad-E- or Ad-T-treated tumors (Fig. 6), suggesting that a stronger in vivo anti-angiogenic effect is obtained when Ad-E is coadministered with Ad-T. Thus, a synergistic antitumor effect against xenograft growth of HCC is achieved through the combined administration of Ad-E and Ad-T.

In the current study, the recombinant Ad-E and Ad-T were sucessfully constructed by means of the adeno-X expression system. Intratumoral administration revealed that a combination treatment employing one-half the dose of Ad-E and Ad-T led to a significant enhanced regression of the tumors compared with treatment with either agent alone. Treated xenografts by combination of Ad-E with Ad-T demonstrated increased apoptosis and reduced angiogenesis in the tumors that may account for the histological observation of tumor growth inhibition. Our findings highlight a promising application prospect in achieving more effective growth inhibition of HCC by means of adenovirus-mediated combinatorial gene therapy.

\section{Acknowledgements}

The authors are grateful for the financial support from the National Natural Science Foundation of China (Grant No. 30900749), the Doctorate Fund of National Education Ministry of China (Grant No. 20090450416) and the Shenzhen Municipal Government and Bureau of Science, Technology and Information through the programs of Shenzhen National Key Lab of Health Science and Technology and the Key Lab of Gene and Antibody Therapy.

\section{References}

1. Cha C, DeMatteo RP and Blumgart LH: Surgery and ablative therapy for hepatocellular carcinoma. J Clin Gastroenterol 35 (Suppl 2): S130-S137, 2002.

2. Forner A, Llovet JM and Bruix J: Hepatocellular carcinoma. Lancet 379: 1245-1255, 2012.

3. El-Serag HB: Hepatocellular carcinoma. N Engl J Med 365: 1118-1127, 2011.

4. Carr BI: Hepatocellular carcinoma: current management and future trends. Gastroenterology 127: S218-S224, 2004

5. Di Maio M, De Maio E, Perrone F, Pignata S and Daniele B: Hepatocellular carcinoma; systemic treatments. J Clin Gastroenterol 35 (Suppl 2): S109-S114, 2002.

6. Prieto J, Qian C, Hernandez-Alcoceba R, GonzalezAseguinolaza G, Mazzolini G, Sangro B and Kramer MG: Gene therapy of liver diseases. Expert Opin Biol Ther 4: 1073-1091, 2004. 
7. Hernández-Alcoceba R, Sangro B and Prieto J: Gene therapy of liver cancer. Ann Hepatol 6: 5-14, 2007.

8. Cosman D: A family of ligands for TNF receptor superfamily. Stem Cells 12: 440-445, 1994.

9. Ashkenazi A and Dixit VM: Death receptors: signaling and modulation. Science 281: 1305-1308, 1998.

10. Walczak H,MillerRE, Ariail K, et al: Tumoricidal activity of tumor necrosis factor-related apoptosis-inducing ligand in vivo. Nat Med 5: 157-163, 1999.

11. Piras V, Hayashi K, Tomita M and Selvarajoo K: Enhancing apoptosis in TRAIL-resistant cancer cells using fundamental response rules. Sci Rep 1: 144, 2011.

12. Yang A, Wilson NS and Ashkenazi A: Proapoptotic DR4 and DR5 signaling in cancer cells: toward clinical translation. Curr Opin Cell Biol 22: 837-844, 2010.

13. Nagane M, Huang HJ and Cavenee WK: The potential of TRAIL for cancer chemotherapy. Apoptosis 6: 191-197, 2001.

14. Yamaguchi N: An analysis of the functional mechanisms of endostatin-the anti-angiogenic activity of endostatin is mediated by its multiple binding ability. Connect Tissue 36: 171-178, 2004

15. O'Reilly MS, Boehm T, Shing Y, et al: Endostatin: an endogenous inhibitor of angiogenesis and tumor growth. Cell 88: 277-285, 1997.

16. Yamaguchi N, Anand-Apte B, Lee M, et al: Endostatin inhibits VEGF-induced endothelial cell migration and tumor growth independently of zinc binding. EMBO J 18: 4414-4423, 1999.

17. Bao Y, Feng WM, Tang CW, Zheng YY, Gong HB and Hou EG: Endostatin inhibits angiogenesis in hepatocellular carcinoma after transarterial chemoembolization. Hepatogastroenterology 59: 1566-1568, 2012.

18. Franceschi RT and Ge C: Gene delivery by adenoviruses. Methods Mol Biol 455: 137-147, 2008.

19. Lee J and Moon C: Current status of experimental therapeutics for head and neck cancer. Exp Biol Med 236: 375-389, 2011.

20. Zhang Y, Qu ZH, Cui M, Guo C, Zhang XM, Ma CH and Sun WS Combined endostatin and TRAIL gene transfer suppresses human hepatocellular carcinoma growth and angiogenesis in nude mice. Cancer Biol Ther 8: 466-473, 2009.

21. Wei YQ, Wang QR, Zhao X, et al: Immunotherapy of tumors with xenogeneic endothelial cells as a vaccine. Nat Med 6 : 1160-1166, 2000.
22. Wei YQ, Huang MJ, Yang L, et al: Immunogene therapy of tumors with vaccine based on Xenopus homologous vascular endothelial growth factor as a model antigen. Proc Natl Acad Sci USA 98: 11545-11550, 2001.

23. Ingber D, Fujita T, Kishimoto S, Sudo K, Kanamaru T, Brem H and Folkman J: Synthetic analogues of fumagillin that inhibit angiogenesis and suppress tumor growth. Nature 348: 555-557, 1990.

24. O'Reilly MS, Holmgren L, Shing Y, et al: Angiostatin: a novel angiogenesis inhibitor that mediates the suppression of metastases by a Lewis lung carcinoma. Cell 79: 315-328, 1994.

25. Sauter BV, Martinet O, Zhang WJ, Mandeli J and Woo SL: Adenovirus-mediated gene transfer of endostatin in vivo results in high level of transgene expression and inhibition of tumor growth and metastases. Proc Natl Acad Sci USA 97: 4802-4807, 2000.

26. Lin T, Zhang L, Davis J, et al: Combination of TRAIL gene therapy and chemotherapy enhances antitumor and antimetastasis effects in chemosensitive and chemoresistant breast cancers. Mol Ther 8: 441-448, 2003.

27. Wissink EH, Verbrugge I, Vink SR, et al: TRAIL enhances efficacy of radiotherapy in a p53 mutant, Bcl-2 overexpressing lymphoid malignancy. Radiother Oncol 80: 214-222, 2006.

28. Wu DS, Wu CM, Huang TH and Xie QD: Combined effects of radiotherapy and endostatin gene therapy in melanoma tumor model. Radiat Environ Biophys 47: 285-291, 2008.

29. Zhu LP, Xing J, Wang QX, et al: Therapeutic efficacy of recombinant human endostatin combined with chemotherapeutics in mice-transplanted tumors. Eur J Pharmacol 617: 23-27, 2009.

30. Kim Y: The effects of nutrient depleted microenvironments and delta-like 1 homologue (DLK1) on apoptosis in neuroblastoma. Nutr Res Pract 4: 455-461, 2010.

31. Braun F, Bertin-Ciftci J, Gallouet AS, Millour J and Juin P: Serum-nutrient starvation induces cell death mediated by Bax and Puma that is counteracted by $\mathrm{p} 21$ and unmasked by Bcl-xL inhibition. PLoS One 6: e23577, 2011. 